\title{
PROTOCOLO DE CUIDADOS DE ENFERMAGEM NO AMBIENTE AEROESPACIAL À PACIENTES TRAUMATIZADOS - CUIDADOS DURANTE E APÓS O VOO ${ }^{1}$
}

\author{
Gabriela Schweitzer², Eliane Regina Pereira do Nascimento ${ }^{3}$, Keyla Cristina do Nascimento ${ }^{4}$, André Ricardo \\ Moreira ${ }^{5}$ Kátia Cilene Godinho Bertoncello ${ }^{6}$
}

\footnotetext{
${ }^{1}$ Recorte da dissertação - Protocolo de cuidados de enfermagem no ambiente aeroespacial à adultos vítimas de trauma: uma pesquisa convergente assistencial", apresentada ao Programa de Pós-Graduação em Enfermagem (PEN) da Universidade Federal de Santa Catarina (UFSC), 2010.

${ }^{2}$ Mestre em Enfermagem. Enfermeira da UTI Terrestre e Aérea do Serviço de Atendimento Móvel de Urgência (SAMU/SC). Santa Catarina, Brasil. E-mail: gabyschw@hotmail.com

${ }^{3}$ Doutora em Enfermagem. Professora Adjunto IV do Departamento de Enfermagem da UFSC. Santa Catarina, Brasil. E-mail: pongopam@terra.com.br

${ }^{4}$ Doutoranda do PEN/UFSC. Bolsista da CAPES. Santa Catarina, Brasil. E-mail: keyla_nascimento@hotmail.com

${ }^{5}$ Mestrando do PEN/UFSC. Enfermeiro da UTI Terrestre e Aérea do SAMU/SC. Santa Catarina, Brasil. E-mail: enfandre@ hotmail.com

${ }^{6}$ Doutora em Enfermagem Fundamental. Professora Adjunto I do Departamento de Enfermagem da UFSC. Santa Catarina, Brasil. E-mail: kbertoncello@yahoo.com.br
}

\begin{abstract}
RESUMO: Este artigo teve como objetivo identificar os cuidados de enfermagem necessários para construir um protocolo de atendimento a pacientes adultos traumatizados, aerorremovidos, específico aos períodos durante e após o voo, baseado nos princípios do Prehospital Trauma Life Support. Trata-se de uma pesquisa qualitativa convergente-assistencial, realizada na Divisão de Operações Aéreas em São José, Santa Catarina, no período de abril a junho de 2010. Para a construção do protocolo foram utilizadas entrevista individual e encontros em grupo. Participaram oito enfermeiros que atuam no serviço. O protocolo contempla cuidados relativos à segurança em voo, avaliação constante do paciente durante a remoção e na entrega ao destino final. O protocolo propiciará uma prática de cuidado mais segura aos pacientes aerorremovidos. Sugere-se outros estudos voltados para a atuação do enfermeiro, como a criação de novos protocolos que orientem a assistência no ambiente aeroespacial.
\end{abstract}

DESCRITORES: Resgate aéreo. Medicina aeroespacial. Enfermagem em emergência. Cuidados de enfermagem. Ferimentos e lesões.

\section{NURSING CARE PROTOCOL FOR TRAUMA PATIENTS IN THE AEROSPACE ENVIRONMENT - DURING AND POST-FLIGHT CARE}

\begin{abstract}
This article aims to identify the nursing care required to build a treatment protocol based on the Prehospital Trauma Life Support principles for adult trauma patients, aero removed, specifically during and post-flight periods. This study is based on convergent-care qualitative research conducted in the Air Operations Division in San Jose, Santa Catarina, Brazil, from April to June, 2010. Individual interviews and group meetings with eight nurses who work in the service were used to construct the protocol. The protocol provides for in-flight safety rules, constant patient evaluation during removal and his/her delivery to their on-ground care destination. The protocol provides safer practical care for aero removed patients. We suggest further study to focus on nursing performance, such as the creation of new protocols that direct aerospace environment nursing care.
\end{abstract}

DESCRIPTORS: Air ambulances. Aerospace medicine. Emergency Nursing. Nursing care. Wounds and injuries.

\section{PROTOCOLO DE ATENCIÓN DE ENFERMERÍA EN EL AMBIENTE AEROESPACIAL A PACIENTES TRAUMATIZADOS - CUIDADOS DURANTE Y DESPUÉS DEL VUELO}

RESUMEN: Este artículo tiene como objetivo identificar los cuidados de enfermería necesarios para crear un protocolo de atención a pacientes adultos traumatizados, aerorremovidos, específico a los períodos durante y después del vuelo, basado en los principios del Prehospital Trauma Life Support. Se trata de una investigación cualitativa convergente llevada a cabo en la División de Operaciones Aéreas en Sao José, Santa Catarina, en el período de abril a junio de 2010. Para la elaboración del protocolo se utilizaron entrevistas individuales y reuniones grupales. Los participantes fueron ocho enfermeros que trabajan en el servicio. El protocolo responde a las reglas de seguridad en vuelo, la evaluación constante del paciente durante la remoción y entrega al destino final. El protocolo proporcionará una práctica más segura para la atención de los pacientes aerorremovidos. Se sugiere otros estudios sobre el desempeño de los enfermeros, tales como la creación de nuevos protocolos que orienten la atención en el entorno aeroespacial.

DESCRIPTORES: Ambulancias aéreas. Medicina aeroespacial. Enfermería de urgencia. Atención de enfermería. Heridas y traumatismos. 


\section{INTRODUÇÃO}

O organismo humano foi feito para viver sobre a crosta terrestre e, portanto, quando embarca numa aeronave para uma viagem, fica sujeito a condições que não são as habituais. Na altitude, ocorre a menor pressão parcial de oxigênio no alvéolo pulmonar, de que resulta a hipóxia. Existe também, a diminuição da pressão atmosférica, que pode levar a problemas de aerodilatação. Existe ainda a turbulência que, como os outros, é fator estressante do voo. E quando submetido a estresse, o organismo humano pode apresentar, além de problemas específicos, os problemas generalizados, devido as alterações psíquicas. ${ }^{1}$

Especializar-se na assistência ao paciente aerorremovido é um desafio para a enfermagem. Esse cuidado de enfermagem especializado só é possível por meio de um estudo sobre fisiologia de voo - que são as respostas do corpo humano as alterações de pressão atmosférica, temperatura e volume, decorrentes do voo e que são fundamentadas nas leis físico-gasosas, determinando como o organismo responde a essas alterações e seus efeitos sobre a tripulação aeroespacial. ${ }^{2}$

O enfermeiro de bordo, que é o profissional de enfermagem que trabalha em aeronaves, ainda é recente em nosso país, tendo poucos cursos específicos na área e na maioria das vezes também tem sua formação voltada para a emergência, com experiência apenas em cuidados a pacientes graves, que se encontram nas emergências e nas Unidades de Terapia Intensivas, falta portanto a vivência de cuidados a pacientes aeroremovidos. ${ }^{3}$

O paciente aerorremovido necessita de profissionais competentes, e o enfermeiro de bordo deve estar preparado para utilizar todos os recursos tecnológicos disponíveis na aeronave para manter o paciente estável durante todo o voo e entregá-lo da melhor maneira possível, com conhecimento científico, principalmente sobre fisiologia de voo. Por isso da importância de uma avaliação constante, seguindo protocolos mundialmente aceitos, como o Prehospital Trauma Life Support (PHTLS). ${ }^{4}$

Esse protocolo orienta a avaliação clínica do paciente e consiste em seguir a sequência do A (Airway - atendimento das vias aéreas e controle de coluna cervical), B (Breathing - respiração), C (Circulation - circulação), D (Disability - incapacidade) e E (Expose - exposição da vítima e proteção do ambiente), que indicam a prioridade do atendimento e direcionam o profissional de saúde, fazendo com que o mesmo não se esqueça e/ou cometa erros na assistência ao paciente, contribuindo para um cuidado mais seguro. ${ }^{5}$

Este modelo de avaliação surgiu porque se notou que o trauma mata, seguindo uma cronologia previsível. Assim, por exemplo, a obstrução das vias aéreas mata mais rapidamente do que a perda da capacidade de respirar, que mata mais rapidamente do que a redução do volume sangüíneo circulante, vindo a seguir o problema mais letal, ou seja, a presença de uma lesão em massa, expansiva, intracraniana. ${ }^{6}$

O PHTLS é um protocolo mundial específico para o atendimento pré-hospitalar, criado para profissionais de saúde de uma maneira em geral - médicos, enfermeiros, paramédicos, socorristas e bombeiros, que atuam no atendimento ao paciente traumatizado fora do ambiente hospitalar. ${ }^{5}$ Logo, toda a questão específica da assistência antes do voo, e os cuidados de enfermagem adaptados neste ambiente não são contemplados neste protocolo.

Cabe ressaltar que se tratando da assistência no ambiente aeroespacial, infelizmente a literatura brasileira ainda é escassa nesta área. A vivência com transporte aeroespacial mostrou a necessidade de adaptar o protocolo de atendimento pré-hospitalar já existente, o PHTLS, para o ambiente aeroespacial.

Diante deste contexto e considerando que o Grupo de Resposta Aérea às Urgências (GRAU) é um serviço muito recente, inédito em Santa Catarina e que não possui ainda qualquer tipo de protocolo de cuidados de enfermagem específico para assistência no helicóptero, este estudo buscou responder a seguinte questão: quais cuidados de enfermagem são necessários para compor um protocolo de cuidados de enfermagem específico do ambiente aeroespacial a pacientes adultos vítimas de trauma?

Para responder esta questão, foi traçado como objetivo: identificar os cuidados de enfermagem necessários para construir um protocolo de atendimento a pacientes adultos vítimas de trauma, no ambiente aeroespacial, fundamentados nos princípios do Prehospital Trauma Life Support, específico aos períodos durante e após o voo de helicóptero.

\section{MÉTODO}

Trata-se de um estudo de abordagem qualitativa que utilizou como método a Pesquisa Convergente-Assistencial (PCA), que tem como característica principal o compromisso de benefi- 
ciar o contexto assistencial, com a intencionalidade de encontrar alternativas para solucionar ou minimizar problemas, realizar mudanças e introduzir inovações na prática. ${ }^{7}$

O estudo foi realizado na base da Divisão de Operações Aéreas (DOA) do Departamento de Polícia Rodoviária Federal (DPRF), localizado na cidade de São José/SC. Trata-se de uma parceria criada através de um acordo entre o Ministério da Justiça via DPRF e Ministério da Saúde via Secretaria do Estado da Saúde/Serviço de Atendimento Móvel de Urgência (SAMU) do Estado de Santa Catarina (SC) em dezembro de 2005. O serviço conta com hangar e um helicóptero modelo Bell 407 configurado com todos os materiais e equipamentos de suporte avançado de vida, tripulado por um comandante/ piloto, um operador de equipamentos especiais, um enfermeiro e um médico. Do total dos nove enfermeiros doSAMU/ SC, oito participaram do estudo.

O projeto de pesquisa foi aprovado pelo Comitê de Ética em Pesquisa da UFSC (Processo $n^{o} 453 / 2009$ ) e teve o consentimento formal das instituições participantes para a coleta de dados. Os participantes foram convidados, informados sobre o objetivo do estudo, assim como acerca das técnicas de coleta que seriam adotadas, todos aceitaram o convite, assinando assim o Termo de Consentimento Livre e Esclarecido.

A coleta de dados foi realizada nos meses de abril a junho de 2010 e ocorreu em duas etapas. A primeira constou de entrevista individual, semiestruturada, com os oito enfermeiros com a seguinte questão norteadora: quais cuidados de enfermagem são necessários para compor um protocolo assistencial específico do ambiente aeroespacial a pacientes adultos vítimas de trauma? Todas as entrevistas foram gravadas com o consentimento prévio dos participantes. Os dados das entrevistas foram organizados conforme a sequência do $A B C$ DE do PHTLS, observando os períodos de cuidado durante e após o voo de helicóptero.

As entrevistas foram realizadas com o intuito de adquirir informações de cada enfermeiro sobre quais cuidados de enfermagem devem constar no protocolo de cuidados, a partir da experiência individual adquirida durante o serviço, qual a prioridade dos cuidados e/ou se o profissional tem ou teve alguma dificuldade no atendimento a esta vítima.

A segunda etapa constou de três encontros em grupo, coordenados pela pesquisadora, que ocorreram em local, dia e hora combinados com os participantes e tiveram em média a duração de três horas.

As discussões em grupo tiveram a finalidade de socializar as informações obtidas nas entrevistas e, a partir disso, em conjunto com os enfermeiros participantes, desenvolver o protocolo de cuidados fundamentado nos princípios do PHTLS.

No primeiro encontro foi apresentado e entregue a cada um dos participantes uma folha impressa, com os resultados das entrevistas, onde foi discutido o protocolo do PHTLS. Esse momento permitiu aos enfermeiros expressarem suas idéias e opiniões, a olhar a situação e identificar carências ou problemas e discrepâncias, como por exemplo, facilidades ou dificuldades na assistência no ambiente aeroespacial. Continuando os trabalhos, foi iniciada a elaboração dos cuidados de enfermagem, onde se construiu uma sequência de atendimento ou de cuidados de enfermagem no ambiente aeroespacial, baseado nos dados das entrevistas e no PHTLS. Todos os participantes anotavam o que estava sendo produzido, na folha entregue no início do encontro.

Nos trinta minutos finais desse encontro, os temas do protocolo: atendimento às vias aéreas, controle da coluna cervical, respiração, circulação, incapacidade, exposição e ambiente e cuidados de enfermagem durante e após voo, foram divididos, por afinidade, entre os enfermeiros participantes. Cada um teve a tarefa de, na reunião seguinte, trazer a justificativa científica relacionada a cada cuidado de enfermagem referente à etapa ou fase do atendimento a que ficou responsável.

O segundo encontro iniciou com a entrega da relação dos cuidados de enfermagem produzidos na reunião anterior e em seguida foi realizada a leitura dos mesmos. Foi dado continuidade à elaboração de mais cuidados e de justificativas. Esse momento serviu também para solucionar dúvidas, fazer ajustes e incluir novos cuidados e suas respectivas justificativas científicas. Foi nesse momento que o protocolo começou a ganhar forma.

Por fim, no terceiro e último encontro, os participantes trouxeram as justificativas e análise de viabilização de cada um dos cuidados de enfermagem, sob sua responsabilidade, concluindo o protocolo. Primeiramente foi realizada uma leitura em conjunto de todo o protocolo de cuidados. $\mathrm{Na}$ medida em que os cuidados e as justificativas eram lidos, ajustes finais iam sendo realizados, bem como a melhora da escrita e da forma. Além disso, foi analisada a viabilidade de cada cuidado e sua justificativa, devido aos condicionamentos 
e limitações que a realidade administrativa do serviço no momento impõe.

\section{RESULTADOS E DISCUSSÃO}

As transformações ocorridas através dos tempos, no atendimento pré-hospitalar, contribuíram para o desenvolvimento de tecnologias complexas e especializadas, como o uso de helicópteros, e que tornaram possível a sobrevivência de pacientes traumatizados, sejam estes muito graves e estando em lugares cada vez mais remotos. Somado a isso, atualmente, há uma preocupação mundial crescente em aliar avanços tecnológicos com uma assistência fundamentada no raciocínio crítico. ${ }^{5}$

Os cuidados de enfermagem durante o voo estão voltados para corrigir e/ou diminuir os efeitos da altitude no organismo, bem como os efeitos das forças gravitacionais e os provocados pelo funcionamento da aeronave. ${ }^{2}$

\section{Protocolo de cuidados de enfermagem durante e após o voo}

No presente estudo é apresentado o protocolo de cuidados de enfermagem elaborado para enfermeiros de bordo atuantes no atendimento a pacientes adultos traumatizados aplicado aos períodos durante e após o voo.

Este protocolo contempla as principais orientações de segurança de voo de helicópteros e a avaliação constante do paciente durante a remoção e na entrega no destino final. Os cuidados estão organizados por meio da sequência do $\mathrm{ABCDE}$, indicando desta forma a prioridade no atendimento e correlacionando-os com a fisiologia de voo. Esses cuidados são seguidos das justificativas científicas que os fundamentam. Apesar de apresentados em uma sequência linear, muitos dos procedimentos podem ser realizados simultaneamente.

Cabe destacar, portanto, que os cuidados realizados antes dos pacientes serem aerorremovidos, como por exemplo, a intubação oro-nasotraqueal e a drenagem de tórax não estão contemplados neste protocolo.

\section{A - Atendimento das vias aéreas e controle da coluna cervical}

- Manter o paciente bem posicionado em decúbito dorsal. Se o trauma for de gestante, deixar a prancha rígida levemente inclinada para a esquerda.
Justificativa: a cabeça, o pescoço, o tronco e a pelve devem ser imobilizados em posição alinhada e neutra, para impedir que qualquer movimento de coluna possa resultar em lesão de medula. Além disso, essa posição facilita a avaliação constante do paciente e minimiza os efeitos das forças gravitacionais decorrentes do voo. ${ }^{8}$

Algumas gestantes podem ter hipotensão supina acentuada. Essa condição pode ocorrer no $3^{\circ}$ trimestre e é causada pela compressão do útero sobre a veia cava inferior. Isso diminui expressivamente o retorno venoso para o coração e, como há menor enchimento do vaso, o débito cardíaco e a pressão arterial diminuem. ${ }^{2}$

\section{B - Respiração}

- Usar filtros bacteriológicos em pacientes intubados, traqueostomizados, em ventilação mecânica ou com reanimador manual.

Justificativa: durante a respiração, há uma perda considerável de vapor d'água para o ar ambiente, principalmente se houver taquipnéia ou se o paciente estiver sob ventilação artificial com ar não umidificado. Os filtros funcionam como umidificadores do oxigênio e como barreira antibacteriana. ${ }^{9-11}$

- Realizar a monitorização da oximetria de pulso e administrar oxigênio sob máscara a todos os pacientes com ventilação espontânea. A fim de prevenir a hipóxia de altitude. Esta é definida como a diminuição da oferta de oxigênio aos tecidos, necessária para o metabolismo celular.

Justificativa: na atmosfera, são identificados quatro níveis que impõem graus progressivos de hipóxia ao organismo com consequentes alterações fisiológicas, dependendo da altitude. Entre 0 e 6.000 pés, pouca ou quase nenhuma alteração fisiológica é observada, exceto no trauma, nos casos de choque e pneumotórax. Sem um mecanismo compensatório como a administração de oxigênio suplementar, a saturação de hemoglobina que é de $98 \%$ no nível do mar cai para $87 \%$ a 10.000 pés e a $60 \%$ a 22.000 pés. $^{2}$

Esses cuidados são particularmente importantes em pacientes com maior risco de descompensação, como nos casos de traumatismo craniano, de grande queimado e de politraumatizados. ${ }^{2-4}$

- Controlar o padrão ventilatório, atentar para a modalidade do ventilador: pressão $x$ volume.

Justificativa: devido às alterações da pressão barométrica decorrentes da altitude, os respirado- 
res volumétricos apresentam aumento do volume corrente, do tempo inspiratório e expiratório, diminuição da frequência das ventilações e alterações imprevisíveis da relação inspiração/expiração. Por isso, os parâmetros ventilatórios devem ser frequentemente reavaliados durante o voo. ${ }^{10-11}$

- Observar sinais de pneumotórax durante o voo.

Justificativa: O aumento da altitude provoca a aerodilatação dos gases, disbarismos. Ficar atento aos sinais clínicos do paciente, pois é praticamente impossível conseguir realizar a ausculta pulmonar durante o voo, devido aos ruídos e vibrações da aeronave. ${ }^{12-14}$

- Manter dreno de tórax aberto.

Justificativa: o paciente está sujeito a alterações da pressão atmosférica com o aumento da altitude, observa-se durante o voo, um possível aumento do pneumotórax e consequentemente um aumento no desconforto respiratório. Estes pacientes podem ter seu quadro de insuficiência respiratória agravado, se forem transportados por helicóptero sem estar com dreno de tórax instalado e funcionante. . $^{8-13}$

Devido à pequena distância existente entre o paciente e o assoalho do helicóptero, deve-se utilizar um sistema de drenagem com válvula unidirecional (válvula de Heimlich), principalmente para se evitar o retorno do conteúdo do frasco de drenagem para dentro da cavidade torácica do paciente, durante as manobras mais bruscas do helicóptero. ${ }^{2-6}$

\section{C - Circulação}

- Monitorizar o paciente com o monitor de multiparâmetros. Este equipamento possibilita o acompanhamento hemodinâmico, contínuo e simultâneo do paciente nos múltiplos parâmetros vitais como: pressão arterial, frequência cardíaca, saturação de oxigênio.

Justificativa: sem esta importante tecnologia, os sinais como a pressão arterial e ausculta pulmonar, não seriam passíveis de mensuração devido ao alto ruído presente no interior do helicóptero, que chega a ultrapassar os 110 decibéis. $^{2}$

- Administrar medicamentos "prescritos como se necessário". Manter soros em pressurizador e medicamentos endovenosos em bomba de seringa. Antes de administrar os medicamentos prescritos como "se necessário", avaliar a presença de sinais clínicos de dor e/ou agitação e de náuseas e/ou êmese.

Justificativa: as soluções parenterais devem ser acondicionadas em seus envólucros próprios e precisam de um pressurizador ou bomba de seringa, para a sua infusão, e para uniformizar o gotejamento da solução. ${ }^{8-14}$

Justificativa: a altitude e suas variações durante o voo dificultam e interferem nas tentativas de infusões endovenosas realizadas por gravidade. Portanto para realizar o este cuidado de forma precisa e segura, o uso desta tecnologia de infusão é fundamental. ${ }^{8-12}$

\section{D - Incapacidade}

- Deixar as medicações de sedação à mão; é clinicamente esperado que o paciente com trauma apresente agitação psicomotora durante o voo.

Justificativa: devido à hipóxia, as alterações no sistema nervoso central podem ocorrer: como a excitação, a agitação psicomotora, a hiperatividade, a inquietação e a euforia. ${ }^{10-12}$

Outro estímulo muito comum durante o voo é a presença de raios solares, que incomodam e ofuscam a visão do paciente, que, somado à sua imobilização na prancha rígida de transporte, resultam em mais um importante fator estressante que gera sofrimento ao paciente e manifestações clínicas como: cefaléia, dor ocular e tonteiras. Portanto, medidas profiláticas são necessárias e são adotadas, dentre elas a farmacológica.

- Atentar para as crises convulsivas. Usar protetor e/ou viseiras.

Justificativa: outro fator importante é o efeito estroboscópico que as pás do rotor principal do helicóptero produzem sobre a visão do paciente, que o estimulam e podem resultar em crises convulsivas. Acalmar o paciente, se necessário, e realizar sedação leve, com o objetivo de tranquilizá-lo e, consequentemente, acalmá-lo. ${ }^{4-6}$

É importante que o paciente consciente seja informado e prevenido sobre a existência de barulhos e de vibrações, que são produzidos pela aeronave, bem como do espaço reduzido em será transportado, onde equipamentos e tripulantes ficarão muito próximos de seu corpo.

São 11 os principais fatores de estresse presentes durante todo o voo aeroespacial: hipóxia, disbarismos, umidade do ar, temperatura, vibrações, ruído, forças acelerativas e gravitacional, luminosidade, sobrecargas musculoesqueléticas, fadiga de voo e fusos horários. ${ }^{1,8-11}$ Estes podem 
repercutir de modo significativo na patologia do paciente, sendo importante adequar a terapêutica de acordo com a condições de voo. , $^{210-11}$

\section{E - Exposição e ambiente}

- Manter o paciente bem posicionado na prancha rígida e com tira tipo aranha. Mantê-lo bem posicionado/alinhado. Justificativa: diminui o desconforto, aumenta a segurança do paciente durante o voo e minimiza os efeitos das forças gravitacionais decorrentes do voo. ${ }^{2}$

- Proteger os ouvidos com abafador de orelha. Ruídos intensos provocam desconforto.

Justificativa: se o paciente estiver lúcido, pode-se colocar o fone de ouvido, com fonia liberada para o mesmo se comunicar com os profissionais. Ruídos acima de 85 decibéis $(\mathrm{dB})$ já são considerados danosos aos ouvidos. Voos longos podem ocasionar em cefaléia, em tonturas, em fadigas, em distúrbios visuais e danos temporários ou permanentes ao ouvido, logo, cabe a prevenção (ruído aeronáutico varia entre $110-130 \mathrm{~dB}){ }^{8-14}$

- Manter o paciente aquecido com manta térmica e cobertor.

Justificativa: é necessário para prevenir a hipotermia. Atentar para a altitude: quando a altitude aumenta, a temperatura diminui. A cada 1.000 pés a temperatura cai $2^{\circ} \mathrm{C}$. Além disso, o paciente, durante o vôo, perde temperatura por convecção - perda de calor devido à ação dos ventos (voar com a porta do helicóptero aberta) e por condução - perda do calor pela proximidade com a fuselagem do helicóptero. ${ }^{2,10-11}$

- Manter os cateteres e as sondas bem fixadas.

Justificativa: para evitar os deslocamentos durante o voo deixá-los abertos, pois os gases podem expandir os cateteres e sondas, com o aumento da altitude. ${ }^{1,8-11}$

- Manter a separação do paciente com a estrutura física da aeronave, interpondo colchonetes de espuma nas laterais.

Justificativa: são medidas importantes para diminuir os efeitos das vibrações sobre o paciente. ${ }^{1,8-11}$

Ao nos tornarmos enfermeiros de atendimento pré-hospitalar, acredita-se que é necessário aceitar a responsabilidade de cuidar do paciente da melhor forma possível, com estudo e dedicação. Durante o atendimento, não há muito tempo para pensar sobre a sequência em que deve ser feita a avaliação do paciente e nem sobre que tratamento deve ter prioridade sobre os outros. Não há tempo para se procurar determinado equipamento ou material dentro da mochila ou da aeronave. Existem também os estressores de voo, como o ruído, a altitude e a vibração. Isso intensifica ainda mais a importância de protocolos de cuidados que orientem o profissional durante a assistência.

\section{Orientações de segurança durante o voo de helicóptero}

- Fixar o soro ou outros equipamentos bem próximos ao paciente e sobre a maca rígida ao levá-lo para a aeronave, e não deixar o lençol, o cobertor ou a manta térmica soltos. Fixá-los com a tira aranha.

Justificativa: ações indispensáveis para maximizar a segurança do voo para a equipe e paciente. Fixar o soro é uma forma de evitar a perda acidental do acesso venoso. Equipamentos devem ser bem fixados para evitar sua queda acidental. Lençóis e mantas térmicas podem "voar" em direção aos rotores da aeronave e causar acidente. ${ }^{2,4-6}$

- Sentar-se no lugar que lhe foi designado ao entrar na aeronave e colocar o fone de ouvido e o cinto de segurança, retirando-os somente com a autorização do piloto.

Justificativa: são medidas importantes e fundamentas para a segurança de voo. Evitar conversar à bordo durante a decolagem e antes do pouso. As conversas podem atrapalhar a concentração do piloto, ou a comunicação com a torre de comando do espaço aéreo nas decolagens e pousos. ${ }^{11-14}$

- Avisar imediatamente o operador de equipamentos especiais ou o piloto ao sentir odor de fumaça, de combustível, ou de algo queimando, e não tomar nenhuma atitude sem autorização.

Justificativa: algum problema com a aeronave pode estar ocorrendo. Estes profissionais são os mais preparados nestas situações. ${ }^{2-5}$

- Comunicar o piloto ao utilizar o celular.

Justificativa: a utilização de telefones celulares dentro do helicóptero é permitida, desde que não interfira na comunicação interna na aeronave. Sempre prestar atenção e evitar conversas durante as decolagens e pousos. As conversas devem ser objetivas para que não se tenha interferência de comunicação. ${ }^{8-13}$

- Manter fixos e bem acondicionados os materiais e equipamentos no interior da aeronave. Devido à vibração, fatores aerodinâmicos e manobras da aeronave. 
Justificativa: reportar imediatamente ao piloto ou ao operador de equipamentos especiais se observar objetos que possam colidir com a aeronave, como pipas, pássaros e fios de alta tensão, por exemplo. Dessa forma, o piloto pode ter tempo de desviar do obstáculo, evitando que o mesmo vá em direção aos rotores, podendo prejudicar o funcionamento do helicóptero e causar acidente. ${ }^{10-13}$

- Obter informações sobre o voo, principalmente o tempo de voo, com o piloto, a fim de programar o cuidado prestado.

Justificativa: o enfermeiro deve ser informado acerca das mudanças de rota de voo/tempo, para planejar o volume de medicação e o consumo de oxigênio, de acordo com o estado clínico do paciente. ${ }^{2-4,8-11}$

- Evitar o uso de brincos, pulseiras, anéis e adereços.

Justificativa: estes objetos podem perfurar ou se prender/desprender, nos fones de ouvido, nos equipamentos em geral, danificando-os e prejudicando o voo. ${ }^{2-3}$

\section{Cuidados de enfermagem após o voo}

- Passar o plantão para o enfermeiro sobre os cuidados de enfermagem realizados.

Justificativa: relatar a cinemática do trauma, o ABCDE, os sinais vitais iniciais, o glasgow, os procedimentos efetuados, as intercorrências, a insuflação do cuff (se foi com água ou ar), em que situação foi feito o acesso venoso, as condições de assepsia para realização das técnicas, o que recebeu de volume, que drogas que foram utilizadas, os curativos/drenos/sondas/cateteres, como foi o transporte, e informar presença ou não de familiar, de retirada de pertences do paciente e de policiais no local. ${ }^{2-5,8-10}$

As informações do pacientes são fundamentais para garantir a continuidade da assistência e da avaliação após o trauma, a fim de minimizar possíveis complicações ou sequelas. ${ }^{2-5}$

- Preencher toda a ficha de atendimento e solicitar a assinatura do médico responsável pelo paciente no hospital.

Justificativa: a ficha de atendimento é considerada como o registro completo das lesões encontradas e das ações do enfermeiro e do médico de bordo. ${ }^{2-4,10-13}$

O PHTLS ancorou o estudo e se mostrou adequado na medida em que norteava as ações do grupo, fundamentando e orientando a cons- trução do protocolo. A sequência do $\mathrm{ABCDE}$ preconizada pelo PHTLS $^{4-5}$ foi imprescindível para o estabelecimento de prioridades de atendimento ao traumatizado aerorremovido e que se tornará um guia diferencial na assistência de enfermagem, porque diferente dos outros protocolos, desenvolveu os cuidados justificados para o durante e após o voo. A justificativa de cada cuidado possibilitou ao profissional o entendimento do porquê de cada cuidado e com isso facilitou o aprendizado e a aquisição de novos conhecimentos.

Desta maneira, a construção do protocolo de cuidados, com intuito de priorizar ou organizar as ações de enfermagem ao paciente traumatizado no ambiente aeroespacial, se torna um importante instrumento na tomada de decisão. Assim sendo, através do protocolo será possível sistematizar a assistência prestada, possibilitando uma avaliação da mesma e orientando as ações necessárias para o cuidado, evitando a repetição de erros. O protocolo servirá também para explicitar o papel do enfermeiro de bordo no cuidado do paciente traumatizado.

\section{CONCLUSÃO}

A assistência de enfermagem prestada no ambiente aeroespacial deve ser embasada principalmente nas alterações fisiológicas decorrentes do voo, utilizando a cientificidade nos cuidados prestados, visando o aperfeiçoamento desta atividade.

A utilização de protocolos de cuidados se torna um importante instrumento na tomada de decisão do enfermeiro de bordo, uma vez que prioriza e organiza as ações de enfermagem ao paciente vítima de trauma, geralmente grave, que requer avaliação constante num ambiente com muitos estressores de voo.

O modo como os cuidados de enfermagem foram apresentados no protocolo seguido de suas justificativas, acredita-se, possibilita ao profissional o entendimento do porquê de cada cuidado e facilitará o aprendizado e a aquisição de novos conhecimentos. Além disso, proporciona uma leitura rápida e sistematizada, estimulando o seu uso pelo enfermeiro, em cada plantão, e não se tornando somente mais um instrumento a ser arquivado.

Sendo assim, reforça-se a importância da utilização do PHTLS para guiar o estudo cientificamente, bem como se destacam outras questões que facilitaram o desenvolvimento deste trabalho: a realização do estudo no local de trabalho das pessoas envolvidas, o entrosamento, o comprometimento 
e a atitude dos enfermeiros participantes, e o fato dos enfermeiros atuarem como profissionais de saúde da realidade em questão, o que possibilitou uma melhor compreensão dos cuidados específicos do ambiente aeroespacial.

A transferência dos resultados não é a de generalizações, e sim, de socialização. Todavia, nada impede que o protocolo construído possa ser transferido para outros cenários/contextos semelhantes. É importante lembrar que este protocolo de cuidados de enfermagem aplica-se apenas para pacientes com trauma, durante e após o voo, e para transportes diurnos, sendo, portanto uma limitação deste estudo, pois o helicóptero deste serviço não realiza voos noturnos, já que exige uma tecnologia toda diferenciada, e também, o protocolo não contempla os importantes cuidados necessários para serem aplicados antes do voo.

Com isso, sugerem-se outros estudos voltados para a atuação do enfermeiro de bordo, como a criação de novos protocolos que orientem a assistência prestada ao paciente aerorremovido, não só a pacientes traumatizados, mas também com outros agravos de saúde e necessidades de cuidados de enfermagem.

\section{REFERÊNCIAS}

1. Pessoa LT. Medicina aviação. Itamaracá (PE): Cristina Publicidade Aérea; 1992.

2. Reis MCF, Vasconcellos DRL, Saiki J, Gentil RC. Os efeitos da fisiologia aérea na assistência de enfermagem ao paciente aerorremovido e na tripulação aeromédica. Acta Paul Enferm. 2000 MaiAgo; 13(2):16-25.

3. Thomaz RR, Miranda MFB, Souza GAG, Gentil RC. Enfermeiro de bordo: uma profissão no ar. Acta Paul Enferm. 1999 Jan-Abr; 12(1):86-96.
4. McSwain NE, Frame S, Salamone JP. PHTLS: Atendimento pré-hospitalar ao traumatizado. $6^{\mathrm{a}}$ ed. Rio de Janeiro (RJ): Elsevier; 2007.

5. Batista SA. Transporte aeromédico. In: Sousa RMC, Calil AM, Paranhos WY, Malvestio MA. Atuação no trauma: uma abordagem para a enfermagem. São Paulo (SP): Atheneu; 2009. p. 493-507.

6. Atzingen MDV, Schimtd DRC, Nonino EAPM. Elaboração e aplicação de um instrumento de avaliação no pós-operatório imediato com base no protocolo do Advanced Trauma Life Suport. Acta Paul. Enferm. 2008 Mai; 21(4):616-23.

7. Trentini M, Paim L. Pesquisa convergenteassistencial: um desenho que une o fazer e o pensar na prática assistencial em saúde-enfermagem. $2^{\mathrm{a}}$ ed. Florianópolis (SC): Insular; 2004.

8. Temporal W. Medicina aeroespacial. Rio de Janeiro (RJ): Luzes; 2005.

9. Pereira Jr GA, Nunes TL, Basile-Filho A. Transporte do paciente crítico. Medicina Ribeirão Preto. 2001 Abr-Jun; 34(2):143-53.

10. Mannarino L, Timerman S. Transporte terrestre e aéreo do paciente crítico. Rev Soc Cardiol Estado São Paulo. 1998; 8(4):866-78.

11. Oliva R. Valoración inicial del paciente com trauma grave. In: Moraza AS, Ayuso DF. Manual de helitransporte sanitario. Barcelona (ES): Elsevier; 2008. p. 203-9.

12. Gentil RC. Aspectos históricos e organizacionais da remoção aeromédica: a dinâmica da assistência de enfermagem. Rev Esc Enferm USP. 1997 Dez; 31(3):452-67.

13. Chapleau W. Manual de emergências: um guia para primeiros socorros. Rio de Janeiro (RJ): Elsevier; 2008.

14. Bueno AA, Bernardes A. Percepção da equipe de enfermagem de um serviço de atendimento pré-hospitalar móvel sobre o gerenciamento de enfermagem. Texto Contexto Enferm. 2010 Jan-Mar; 19(1):45-53. 